\title{
Pattern formation in stiff oscillatory media with nonlocal coupling: A numerical study of the hydrogen oxidation reaction on Pt electrodes in the presence of poisons
}

\author{
F. Plenge, ${ }^{1} \mathrm{H}$. Varela, ${ }^{1, *}$ and K. Krischer ${ }^{2, \dagger}$ \\ ${ }^{1}$ Fritz-Haber Institute of the Max-Planck Society, Faradayweg 4-6, 14195 Berlin, Germany \\ ${ }^{2}$ Physik Department E19, TU München, James-Franck-Str. 1, 85748 Garching, Germany
}

(Received 17 June 2005; published 21 December 2005)

\begin{abstract}
The impact of the strength of negative (desynchronizing) global coupling (NGC) on the spatiotemporal dynamics of an electrochemical relaxation oscillator is studied numerically with a prototypical model, the electro-oxidation of hydrogen in the presence of poisons. The results are compared with recent experiments. The NGC has a destabilizing effect on the homogeneous oscillations. Both, in theory and in experiments, the basic patterns found with increasing global coupling strength are modulated oscillations, target patterns (including an asymmetric variant), and modulated pulses, the average spatial inhomogeneity during an oscillation increasing with the intensity of the NGC. It is suggested that this scenario is typical for strong relaxation oscillations, and a comparison with an electrochemical oscillator exhibiting harmonic oscillations points to the fact that the critical coupling strength, upon which the complete synchronization is destroyed, is larger for relaxation oscillations than for harmonic oscillations. In addition, the numerical simulations predicted two- and three-phase cluster patterns at high coupling strength. Also in experiments cluster patterns were observed, however only in parameter regions of the local dynamics which were different from the one investigated in this study.
\end{abstract}

DOI: 10.1103/PhysRevE.72.066211

\section{INTRODUCTION}

Active media often possess two types of spatial interactions, local, or, to be more general, nonlocal interactions with the strength of the spatial coupling decreasing strongly with the distance from a reference point, and global interactions by which all locations are coupled to each other with the same strength. Examples include surface reactions under low pressure [1-3]. Here adsorbed species diffuse on the surface thereby locally coupling adjacent locations, while the gas phase mixes rapidly and thus the exchange of reactants with the gas phase globally couples all reaction sites. Other examples are systems that are controled through an external electrical circuit, such as semiconductor devices [4], gas discharge tubes [5], or electrochemical systems [6]. In these systems, the spatially extended device, i.e., the interior system (the semiconductor element in the first case, and electrodes in the latter two cases), is connected to a voltage or current generator through an external circuit. Some transport process along the extended device couples positions locally and any Ohmic resistance in the external circuit introduces a global feedback in the voltage drop across the interior system which is felt at all positions of the spatially extended device with equal intensity. In a similar manner, a global coupling (GC) is introduced in catalytic systems operated at high pressure via electric heating of the catalyst $[7,8]$. Also in biological systems the combined action of local and global interaction is often decisive for the dynamics. Cardiac cells are

\footnotetext{
*Present address: Instituto de Quimica de Sao Carlos-USP, Sao Carlos-SP, Brasil

†Electronic address: krischer@ph.tum.de; URL: http:// www.physik.tu-muenchen.de/lehrstuehle/E19/krischer.html
}

PACS number(s): 82.40.Np, 82.45.-h, 05.45.-a, 05.65.+b

electrically connected by resistive pores and globally entrained by signals generated at the sinoatrial node [9], and neural tissues often exhibit local and global interactions [10], to mention just two examples. A global feedback can also be deliberately imposed on a distributed system to manipulate or control its spatiotemporal dynamics, as was demonstrated with the light-sensitive Belousov-Zhabotinsky (BZ) reaction [11] and the $\mathrm{CO}$ oxidation on $\mathrm{Pt}(110)$ under low pressure [12].

Many of the above-mentioned systems can be modeled by a set of reaction-diffusion equations of the following form:

$$
\frac{\partial c_{i}(r, t)}{\partial t}=f_{i}\left(c_{i}, c_{j}\right)+\alpha\left[\left\langle c_{i}\right\rangle-c_{i}(r)\right]+D_{i} \Delta c_{i},
$$

where the nonlinear functions $f_{i}$ describe the local dynamics of the variables $c_{i}$. The second term takes into account the global coupling; the cornered brackets show that the spatial average is taken, and $\alpha$ is the constant global coupling strength. The third term describes simple diffusion with the diffusion coefficient $D_{i}$. In a more general formulation allowing also for nonlocal coupling as occurring in the electrochemical systems considered here, the diffusion term has to be replaced by an appropriate generalized coupling term, such as an integral over a coupling function $H$ [13-15]. In electrochemical systems the coupling function $H$ is strictly positive, and thus the nonlocal coupling is synchronizing, as is the spatial coupling through diffusion. In contrast, the strength of the global coupling $\alpha$ might be positive or negative, and thus the global coupling can have synchronizing or desynchronizing properties, respectively.

The impact of the global coupling in reaction-diffusion systems has been intensively studied for different active media, and the fundamental states induced by global coupling 
are well known. When the local dynamics is bistable, the basic action of the GC is to drive the system into two stationary domains with distinct properties. Homogeneous stationary states can be destabilized through a local bifurcation where the first nontrivial mode becomes unstable involving either a real or a complex eigenvalue. In the first case a stationary sinusoidal profile with the wave number $k=1$ develops; the second one corresponds to a wave bifurcation with $k=1$, leading to standing waves or pulses. In the oscillatory region, the GC favors the formation of oscillatory clusters, i.e., spatial regions within which all positions oscillate with the same amplitude and frequency, whereas different cluster regions oscillate with a constant phase difference and possibly also different amplitudes. Yet, the diversity of GC-induced patterns is by far richer. Not only do the basic states undergo a variety of secondary bifurcations, but, depending on the coupling parameters and the properties of the local dynamics, the basic patterns might also be altered drastically or even replaced by completely different dynamical states.

So far there are only comparatively few systematic studies that shed light onto the variation of the patterns with the GC strength. In this paper we present a detailed numerical study of the dynamics of a globally coupled, electrochemical relaxation oscillator as a function of the GC strength $\alpha$ and the applied voltage $U . U$ is the main bifurcation parameter in electrochemical systems; essentially, it controls the distance from the equilibrium potential of the main reaction. This investigation was motivated by experiments on the dynamics of the hydrogen oxidation reaction (HOR) on Pt in the presence of poisons, which revealed a rich variety of partly unknown patterns for a sufficiently large negative GC strength, among them target patterns, a dynamical state termed as an asymmetric target pattern, and mixed states between these two patterns as well as modulated pulses [16].

The HOR oscillator is a prototypical electrochemical oscillator whose local dynamics is well understood [17]. It involves the adsorption and desorption of two species, in our case $\mathrm{Cu}^{2+}$ ions and $\mathrm{Cl}^{-}$ions which both inhibit oxidation of hydrogen when adsorbed, i.e., they poison the electrode for hydrogen oxidation. Owing to the opposite potential dependence of the adsorption of the two species, one of them, $\mathrm{Cl}^{-}$ takes part in the positive feedback loop, whereas $\mathrm{Cu}^{2+}$ is an essential species of the inhibitory, negative feedback loop. Different sites of the electrode are coupled by (nonlocal) migration coupling and by GC arising from the potentiostatic control of the reaction [6]. When induced by the potentiostatic operation mode, the GC is desynchronizing or negative. The local dynamics of the HOR oscillator is captured with a four variable model nearly quantitatively. The spatiotemporal model employed in the present simulation is based on this homogeneous model and is introduced in Sec. II. An overview of the observed spatiotemporal patterns at different values of the above parameters is presented in Sec. III A. The properties of the most significant patterns are explored in the following subsections. The results are put into a general frame and compared with experiments in Sec. IV, and Sec. V summarizes the presented findings.

\section{MODEL}

The local dynamics of the HOR in the presence of poisons is captured nearly quantitatively with a reduced four variable model, the variables being the double layer potential, $\phi_{\mathrm{DL}}$, the coverages of $\mathrm{Cu}, \theta_{\mathrm{Cu}}$, and $\mathrm{Cl}^{-}, \theta_{\mathrm{Cl}}$, and the concentration of $\mathrm{Cu}^{2+}$ ions in the reaction plane, $c$ [17]. The model describing the spatiotemporal dynamics is obtained taking additionally into account the spatial coupling mediated by the electric field within the electrolyte (the migration coupling) and the NGC arising from the potentiostatic operation mode whenever the reference electrode (RE) is close to the working electrode (WE) [18]. Both coupling terms enter the evolution equation of $\phi_{\mathrm{DL}}$, which plays an activatory role in the system. Compared to migration coupling, spatial coupling through diffusion of the two adsorbates or of $\mathrm{Cu}^{2+}$ ions in the reaction plane is negligible and, as was tested in simulations, does not alter the dynamics. Consequently, it is not considered in the simulations, and the dimensionless model of the spatiotemporal dynamics reads

$$
\begin{gathered}
\dot{\phi_{\mathrm{DL}}}=-i_{r}+\frac{\sigma}{\beta}\left(U-\phi_{\mathrm{DL}}\right)+\frac{\sigma \rho}{\beta}\left(\left\langle\phi_{\mathrm{DL}}\right\rangle-\phi_{\mathrm{DL}}\right) \\
-\left.\frac{\sigma(1+\rho)}{\beta}\left(\frac{\partial \phi}{\partial z}+\phi\right)\right|_{0} \\
\tau_{\mathrm{Cl}} \dot{\theta}_{\mathrm{Cl}}=\left(1+\chi \theta_{\mathrm{Cu}}\right)\left(1-\theta_{\mathrm{Cu}}-\theta_{\mathrm{Cl}}\right) e^{\phi_{\mathrm{DL}}}-p_{\mathrm{Cl}} \theta_{\mathrm{Cl}} e^{-\phi_{\mathrm{DL}}}, \\
\tau_{\mathrm{Cu}} \dot{\theta}_{\mathrm{Cu}}=v_{\mathrm{Cu}}^{a}-v_{\mathrm{Cu}}^{d}, \\
\dot{c}_{\mathrm{Cu}}=1-c_{\mathrm{Cu}}-\nu \tau_{\mathrm{Cu}}^{-1}\left(v_{\mathrm{Cu}}^{a}-v_{\mathrm{Cu}}^{d}\right),
\end{gathered}
$$

with

$$
\begin{gathered}
v_{\mathrm{Cu}}^{a}-v_{\mathrm{Cu}}^{d}=c_{\mathrm{Cu}}\left(1-\theta_{\mathrm{Cu}}-\theta_{\mathrm{Cl}}\right) e^{-a_{\mathrm{Cu}} \phi_{\mathrm{DL}}}-p_{\mathrm{Cu}} \theta_{\mathrm{Cu}} e^{a_{\mathrm{Cu}} \phi_{\mathrm{DL}}}, \\
i_{r}=c_{1}\left(1-\theta_{\mathrm{Cu}}-\theta_{\mathrm{Cl}}\right)\left[1-2\left(1+e^{c_{2} \phi_{\mathrm{DL}}}\right)^{-1}\right] .
\end{gathered}
$$

The transformations into physical quantities and the definitions of the dimensionless parameters are given in the Appendix.

The first two terms of Eq. (1) describe the current density of hydrogen oxidation, $i_{r}$, and the migration current density that would flow in a homogeneous situation, respectively. They, thus, comprise the contribution of the local dynamics to the evolution of $\phi_{\mathrm{DL}}$. The reaction current is fitted to the theoretically derived data using a more extensive model [17]; the fit parameters $c_{1}$ and $c_{2}$ are given in Table I. $\beta / \sigma$ represents the dimensionless resistance between the WE and the RE. The third term describes the global coupling. The cornered brackets again indicate that the spatial average is taken, and $\rho \in]-1,0]$ determines the strength of the negative GC. Physically $\rho$ depends on the ratio of two resistances, the resistance between the RE and the WE and the one between the WE and the counter-electrode (CE), and is thus experimentally well accessible. The last term in Eq. (1), finally, describes the nonlocal migration coupling. $z$ is the direction perpendicular to the WE and $z=0$ is a location at the WE. The aspect ratio $\beta$ determines the range of the coupling 
TABLE I. Values of the nondimensional parameters used if not stated otherwise. (Note that the number of digits reflects the computer precision.) The values are calculated from realistic, dimensional values of the physical parameters compiled in [17] according to the equations given in the Appendix.

\begin{tabular}{lc}
\hline \hline$\sigma=0.8368950770951986$ & $\beta=2.827433388230814$ \\
$\tau_{\mathrm{Cl}}=83760.0902552427$ & $\tau_{\mathrm{Cu}}=21.03215159159541$ \\
$p_{\mathrm{Cl}}=497.8642409711874$ & $p_{\mathrm{Cu}}=p_{\mathrm{Cu}}^{0}:=3.13913279204803 \times 10^{-11}$ \\
$\nu=17401.49196751466$ & $c_{1}=4.89745966763657$ \\
$c_{2}=39.553360429673866$ & $a_{\mathrm{Cu}}=38 / 3$ \\
\hline \hline
\end{tabular}

[19], and $\sigma(1+\rho)$ is the dimensionless conductivity. Note that in order to separate the global coupling term from the local part of the dynamics, the dimensionless $\sigma$ also depends on $\rho$ (see Appendix). Thus, changing $\rho$ leaves the homogeneous states unchanged on the expense of changing at the same time the conductivity. A variation of $\rho$ at constant conductivity, on the other hand, requires that $\sigma$ is changed, altering thus the homogeneous states. The latter situation is realized in experiments, whereas the former one helps elucidate the role the global coupling plays for the dynamics. Hence, below both types of parameter variations are considered.

The migration coupling is expressed in terms of the potential in the electrolyte, $\phi$. Since Laplace's equation is valid in the entire electrolyte $(\Delta \phi=0)$ and potential variations at the CE (located at $z=1$ ) can be neglected, $\left.\phi\right|_{z=1}$ can be set to zero, and $\phi_{\mathrm{DL}}=U-\left.\phi\right|_{z=0}$ is the only time-dependent boundary condition of Laplace's equation. Thus, Eq. (1) is a closed expression.

The evolution of $\theta_{\mathrm{Cu}}$ and $\theta_{\mathrm{Cl}}$ [Eqs. (2) and (3)] are governed by the adsorption and desorption rates, respectively. $\tau_{\mathrm{Cl}}$ and $\tau_{\mathrm{Cu}}$ denote the dimensionless relaxation times of the activatory coverage $\theta_{\mathrm{Cl}}$ and the inhibitory coverage $\theta_{\mathrm{Cu}}$, respectively. $\chi$ is the only free parameter introduced to model the reported enhanced adsorption rate of $\mathrm{Cl}^{-}$in the presence of adsorbed $\mathrm{Cu}$ [20]. $a_{\mathrm{Cu}}$ governs the potential dependence of $\mathrm{Cu}$ adsorption and desorption and $p_{\mathrm{Cl}, \mathrm{Cu}}$ determine the adsorption and/or desorption equilibrium potential of $\mathrm{Cu}$ and $\mathrm{Cl}^{-}$, respectively.

The change of $c_{\mathrm{Cu}}$ [Eq. (4)] results from the difference between the diffusion fluxes to and from the bulk electrolyte solution, on the one hand, and adsorption and desorption fluxes at the electrode, on the other hand. $\nu$ defines the relative speed of adsorption and desorption of $\mathrm{Cu}^{2+}$ ions with respect to diffusion from the bulk to the reaction plane.

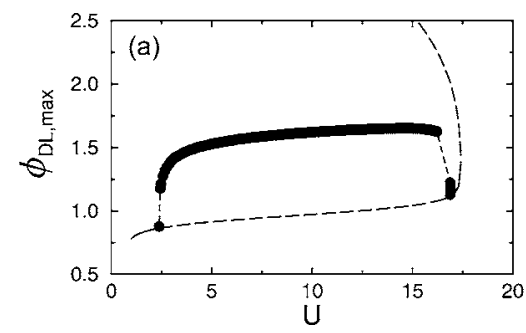

Since the experiments were done with a thin ring electrode on which patterns form only along the azimuthal direction, simulations are carried out along the one-dimensional (1D) electrode, and periodic boundary conditions are used. The electrolyte was restricted to a two-dimensional cylindrical surface bound by the WE at one end and the CE at the other end. For this geometry the eigenfunctions of the Laplace operator along the azimuthal direction are Fourier functions [21]. Expanding all variables in a Fourier series, evolution equations for the Fourier coefficients are obtained. The resulting set of ordinary differential equations was integrated using lsode [22] with a relative and absolute tolerance of $10^{-14}$. Between 31 and 127 Fourier modes were used, and different initial conditions were employed: In most cases, a state close to the homogeneous (unstable) limit cycle with noise of a relative amplitude $<10^{-4}$ superimposed was chosen as initial condition. This comes closest to the initial conditions in experiments. To test for the coexistence of patterns, initial conditions with a nearly rectangular perturbation in space were employed, both with all variables in phase and with a phase mismatch in the individual variables. Furthermore, also solutions obtained at parameter values close by were used as initial conditions. Note, however, that owing to the large phase space and the long integration time it was not possible to perform an exhaustive study and we cannot exclude that further stable patterns exist in the parameter regions investigated.

The values of the nondimensional parameters as obtained from experimental values and constants compiled in [17] are given in Table I. $\tau_{\mathrm{Cl}}, \tau_{\mathrm{Cu}}, p_{\mathrm{Cl}}, \nu, c_{1}, c_{2}$, and $a_{\mathrm{Cu}}$ are fixed at the given values throughout this section. The main bifurcation parameters are the applied voltage, $U$, and the global coupling strength, $\rho$. The homogeneous steady states and the maximum amplitude of oscillatory solutions of $\phi_{\mathrm{DL}}$ are shown as a function of $U$ in Fig. 1(a).

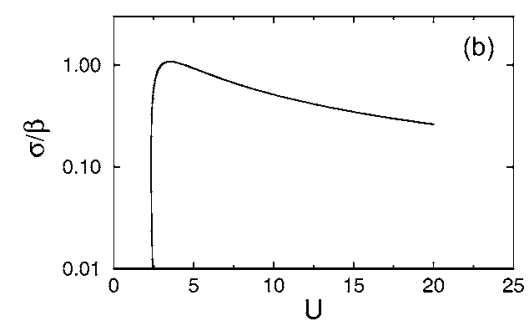

FIG. 1. (a) One parameter continuation of the homogeneous steady states and homogeneous periodic solutions for the dimensionless model, Eqs. (1)-(4). Solid line and dashed lines, stable and unstable stationary steady state, respectively; dots, maximal amplitude of homogeneous periodic solutions (stable or unstable depending on $\rho$, s.b.). For parameters see Table I, $\sigma / \beta \approx 0.296$. (b) Location of Hopf bifurcation points in the $(\sigma / \beta-U)$-parameter plane for the same parameter set. 


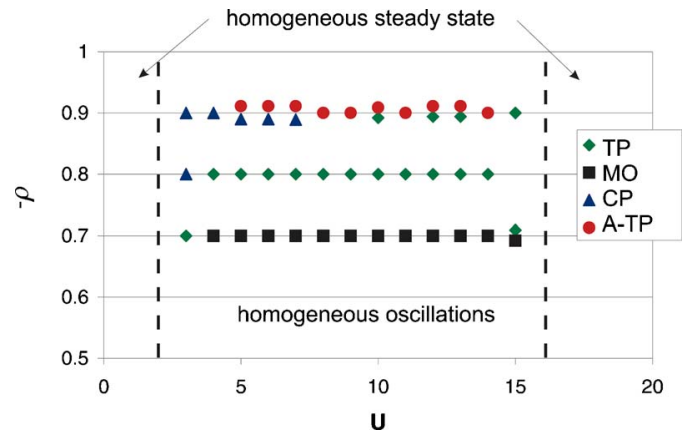

FIG. 2. (Color online) Spatiotemporal patterns in the $U$ $\rho$-parameter plane for $p_{\mathrm{Cu}}=p_{\mathrm{Cu}}^{0} \times 10^{-4}$ (for other parameters see Table I). The homogeneous steady state is observed on the left and right borders of the figure (dashed lines) and homogeneous oscillations are found for $\rho \geqslant-0.6$. Bistability is indicated by stacking the respective symbols. TP, target pattern; MO, modulated oscillations; $\mathrm{CP}$, cluster pattern; A-TP, asymmetric target pattern.

At both ends the oscillatory region is encircled by supercritical Hopf bifurcations. As mentioned above, also $\sigma$ is varied in the following sections to be able to compare systems with identical physical conductivity under different global couplings. The continuation of the two Hopf bifurcations present for the parameter values of Table I [cf. Fig. 1(a)] is presented in Fig. 1(b) in the $(\sigma / \beta-U)$-plane. The point of the Hopf bifurcation at lower $U$, i.e. lower $i_{r}$, is almost independent of $\sigma / \beta$ in a wide $\sigma / \beta$-interval. At higher $U$ the limit cycle is destroyed via a second Hopf bifurcation for high conductivities, cf. Fig. 1(a), and via a saddle-loop bifurcation involving the coexisting saddle fixed point present at higher $\phi_{\mathrm{DL}}$ for lower $\sigma / \beta$ [not shown in Fig. 1(b), cf. upper right corner in Fig. 1(a)].

Additionally, the aspect ratio $\beta$ and $p_{\mathrm{Cu}}$ are varied. $p_{\mathrm{Cu}}$ essentially determines the bulk concentration of copper which has proved to be an important parameter in experiments.

Equations (1)-(4) represent a stiff dynamical system for physically meaningful parameters as given in Table I. For this reason continuations of the spatially extended system proved to be not feasible. Furthermore, due to long integration times, simulations could only be carried out for a limited number of points in parameter space. Integrations were performed until transients decayed. Typically about 50 oscillation periods of the homogeneous system sufficed to observe stable spatiotemporal behavior.

\section{RESULTS}

\section{A. Overview of observed patterns}

The spatiotemporal dynamics of the HOR in the presence of $\mathrm{Cl}^{-}$and $\mathrm{Cu}^{2+}$ was investigated on a grid over the two main bifurcation parameters, $U$ and $\rho$, using model Eqs. (1)-(4) and parameter values given in Table I if not stated otherwise. $U$ was varied in the oscillatory region of the HOR and $\rho$ values were chosen in the NGC interval $\rho \in]-1,0]$. The parameter grid was evaluated at three different values of $p_{\mathrm{Cu}}$ and the results are summarized in Figs. 2, 3, and 4.

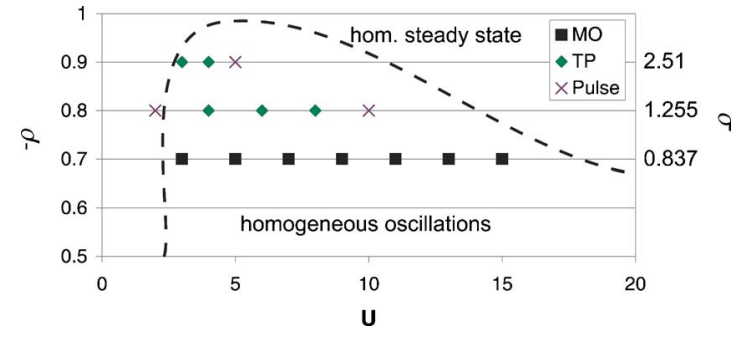

FIG. 3. (Color online) Pattern formation under varying global coupling $\rho$ and adjusting $\sigma$ at the same time to investigate the system behavior for fixed physical conductivity $[\sigma(1+\rho)=$ const $]$. The homogeneous steady state is globally stable outside the region marked with the dashed line (cf. Fig. 1). $p_{\mathrm{Cu}}=p_{\mathrm{Cu}}^{0} \times 10^{-2}$, for other parameters see Table I. MO, modulated oscillations; TP, target pattern.

Simulations without global coupling yield homogeneous dynamics for aspect ratios $\beta$ of the order of 1 . Systems with smaller aspect ratios also display pulses. Homogeneous oscillations stay stable up to $\rho \approx-0.65$ for $\beta \simeq 2.8$. For $U$ $\lesssim 2.3$ and $U \gtrsim 17.8$ the system relaxes to the homogeneous stationary fixed point, cf. Fig. 1. For $U$ in the oscillatory interval and $\rho \lessgtr-0.65$ a variety of spatiotemporal patterns are observed, which are characterized in detail in the following sections. They include pulses, antiphase oscillations, modulated oscillations (MO), so-called cluster patterns (CP), one dimensional target patterns (TP) (i.e., pulse pairs which are periodically emitted from an oscillatory wave source in analogy to target patterns observed in two-dimensional systems) and patterns that were termed asymmetric target patterns (A-TP) [16]. Several parameter combinations support multiple spatiotemporal patterns such that the final pattern depends on the initial condition (cf. Fig. 2).

Figure 2 summarizes the findings for $p_{\mathrm{Cu}}=p_{\mathrm{Cu}}^{0} \times 10^{-4}$ and thus corresponds to the lowest investigated copper concentration. The behavior in this region proved to be the richest one, especially for strong negative global coupling, $\rho=-0.9$.

In Fig. 3 pattern formation as a function of the applied potential and the global coupling was investigated for a fixed physical conductivity at $p_{\mathrm{Cu}}=p_{\mathrm{Cu}}^{0} \times 10^{-2}$. Consequently, $\sigma$ had to be changed for different values of $\rho$. Ergo, also the homogeneous dynamics and hence the $U$ range where oscil-

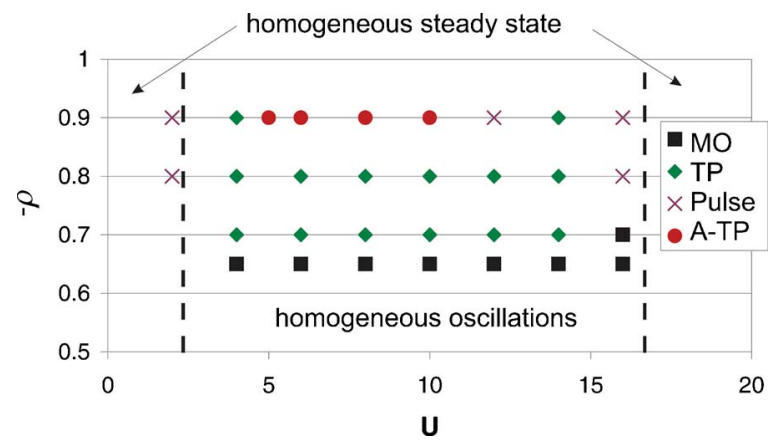

FIG. 4. (Color online) Approximate regions where various patterns induced by the negative global coupling are observed for $p_{\mathrm{Cu}}=p_{\mathrm{Cu}}^{0}$ (for other parameters see Table I). MO, modulated oscillations; TP, target pattern; A-TP, asymmetric target pattern. 


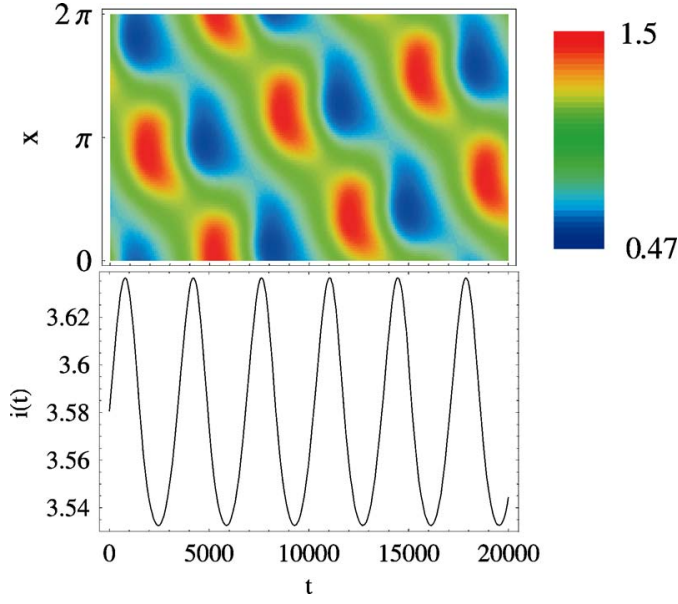

FIG. 5. (Color online) Rotating antiphase oscillation. Upper plate, $\phi_{\mathrm{DL}}(x, t)$; lower plate, $i(t)$. Parameters: $U=5, \sigma=2.51069$, $p_{\mathrm{Cu}}=p_{\mathrm{Cu}}^{0}, \rho=-0.7$. The map used here and in the following is also displayed with minimum and maximum values of $\phi_{\mathrm{DL}}$ (though $\phi_{\mathrm{DL}, \text { min }}$ and $\phi_{\mathrm{DL}, \text { max }}$ differ in the individual figures). For other parameters see Table I.

lations could be observed at all change with changing global coupling (cf. Fig. 1).

The observed dynamics for $p_{\mathrm{Cu}}=p_{\mathrm{Cu}}^{0}$ are displayed in Fig. 4. It corresponds to parameter values investigated in the previous section dealing with the homogeneous dynamics (cf. Fig. 1).

A few general trends apart from the mere existence region of inhomogeneous spatiotemporal behavior can be observed in Figs. 2-4. Pulses are favored at the lower and upper $U$ boundary of oscillatory behavior. Pulses are also observed for $U$ values outside the oscillatory region at the lower $U$ boundary. Thus, these pulses indicate that either excitable dynamics exist in a small parameter region before the Hopf bifurcation or that the wave instability destabilizes the homogeneous steady state at lower $U$. A similar behavior is not exhibited at the upper $U$ boundary. Modulated oscillations and target patterns are found for lower values of the negative global coupling. The richest behavior is displayed at $\rho=-0.9$ where asymmetric target patterns, cluster patterns, and multistability between those and other aforementioned patterns exist.

In the next sections characteristics and different forms of occurrence of the above-mentioned patterns are studied in detail.

\section{B. Pulses and antiphase oscillations}

As mentioned above the most basic patterns to be expected in an oscillatory system under NGC, namely, pulses and antiphase oscillations, are only observed near the boundaries of oscillatory behavior. In both cases, however, the simplest form of these patterns was hardly observed at all. Instead, the antiphase oscillations were found to be translatory unstable, i.e., the entire pattern rotates around the ring as depicted in Fig. 5. A common characteristic of the observed pulses is that they exhibit an oscillatory instability resulting in breathing of the pulse width and a nonconstant pulse speed. These pulses are usually termed modulated pulses. After the wave bifurcation giving rise to traveling wave solutions, modulated pulses stem from a second oscillatory instability introducing a second frequency into the system. This is apparent in the time series of the total current density that oscillates with a frequency which is independent from the circulation time of the pulse.

Pulses during oscillatory oxidation of hydrogen under NGC always display apparently chaotic modulations resulting in a chaotic total current density, see Fig. 6(a) [33]. These chaotically modulated pulses coexist with asymmetric target patterns in certain parameter regions (s.b.).

Pulses traveling with constant shape and speed are found only in the regime in which the mode of homogeneous oscillations is not yet present in the system and for high $p_{\mathrm{Cu}}$. The same is true for modulated pulses displaying a periodic modulation. The modulation amplitude of the periodically modulated pulse is strongly decreased as compared to the chaotic situation, cf. Fig. 6.

\section{Modulated oscillations (MOs) and target patterns (TPs)}

The most common patterns displayed by Eqs. (1)-(4) in the region of intermediate NGC are modulated oscillations and target patterns (cf. Figs. 2-4). An example of a modulated oscillation is depicted in Fig. 7. The pattern is practically homogeneous apart from the fast stages of the oscillations. The inhomogeneous part of the spatiotemporal motion can be made visible by subtracting the space averaged value of $\phi_{\mathrm{DL}}$ at every time moment, see Fig. 7(b). It becomes clear that the homogeneous oscillations are modulated with a spatial wave number one which only shows up on the fast transitions connecting active and passive state. The representation of the dynamics as in Fig. 7 , i.e., the $(x, t)$ plot of $\phi_{\mathrm{DL}}$

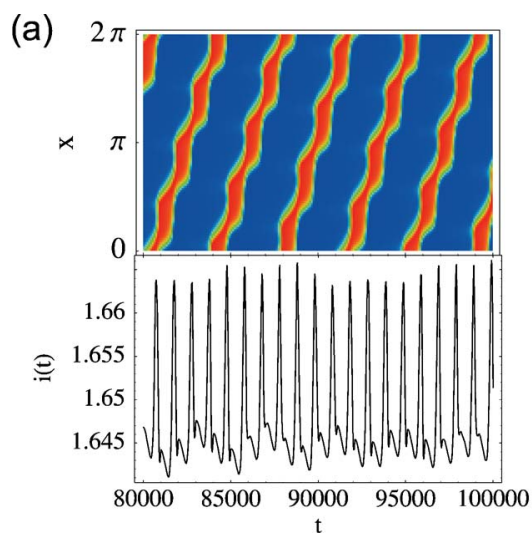

FIG. 6. (Color online) Typical pulse forms observed in the (a) oscillatory and (b) nonoscillatory regime. A second independent frequency is visible in the total current density. Upper plates, $\phi_{\mathrm{DL}}(x, t)$; lower plates, $i(t)$. (a) Chaotically modulated pulse. Parameters: $U=6, p_{\mathrm{Cu}}=p_{\mathrm{Cu}}^{0}$, $\rho=-0.9$. (b) Periodically modulated pulse. Parameters: $U=2, \sigma=1.125534, p_{\mathrm{Cu}}=p_{\mathrm{Cu}}^{0} \times 10^{-2}$, $\rho=-0.9$. For other parameters see Table I. 
(a)

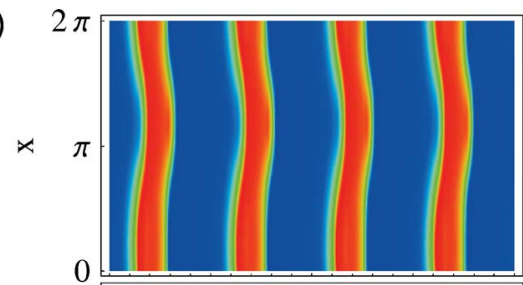

(b)

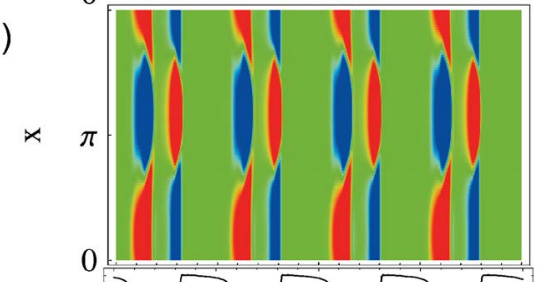

(c)

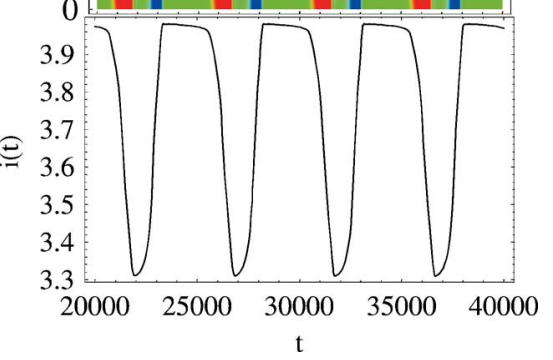

FIG. 7. (Color online) Modulated oscillations. (a) $\phi_{\mathrm{DL}}(x, t)$; (b) Inhomogeneous part of the double layer potential, $\phi_{\mathrm{DL}}-\left\langle\phi_{\mathrm{DL}}\right\rangle$; (c) Total current density as a function of time displaying the relaxationlike behavior of the underlying oscillations. Parameters: $U=14$, $p_{\mathrm{Cu}}=p_{\mathrm{Cu}}^{0} \times 10^{-4}, \rho=-0.7$, for other parameters see Table I.

and $\phi_{\mathrm{DL}}-\left\langle\phi_{\mathrm{DL}}\right\rangle$ supplemented with the total current density as a function of time, is used in all plots from now on.

Modulated oscillations are observed for low NGC strengths, i.e., the desynchronizing effect is not effective enough to promote inhomogeneities at all times. The "pumping" into inhomogeneous modes is strong enough to promote spatial patterns only during the fast stages of the relaxation oscillations. Modulated oscillations are thus genuine patterns to observe in systems under NGC displaying relaxationlike oscillations in the homogeneous system.
Increasing the NGC, the inhomogeneities become more pronounced until the passive stage of the oscillation is completely inhomogeneous. Such a pattern is shown in Fig. 8(a). It apparently resembles a 1D target pattern. Two waves are emitted from a source point and travel along the ring until they annihilate on the opposite side of the ring [34]. The waves are decelerated which yields an asymmetric pattern in time and facilitates the distinction between target patterns and modulated oscillations. To shed light on the question of whether the NGC or the nonlocal migration coupling is responsible for the deceleration, the aspect ratio $\beta$ is decreased to values where the migration coupling can be approximated by a diffusional term [23]. The resulting target pattern is displayed in Fig. 8(b) and the constant speed of the traveling waves suggests that the migration coupling causes the deceleration. Note however that the global coupling strength in Fig. 8(b) is decreased compared to Fig. 8(a).

A further apparent difference between modulated oscillations and target patterns is the joining of the inhomogeneities in the uprising and falling flanks that is clearly absent in the subtracted data of the modulated oscillation shown in Fig. 7. However, the transition from modulated oscillations to target patterns is continuous. A suitable quantitative measure to decide whether a pattern should be classified as a modulated oscillation or a target pattern is the time average over one oscillation of the total current density. Target patterns exhibit a more pronounced spatial symmetry breaking. On average the total current density in the region in which the two waves meet is higher than on the rest of the ring. Also modulated oscillations show some asymmetry, but less pronounced than target patterns. Thus, a threshold $r_{t}$ on the basis of the maximum of

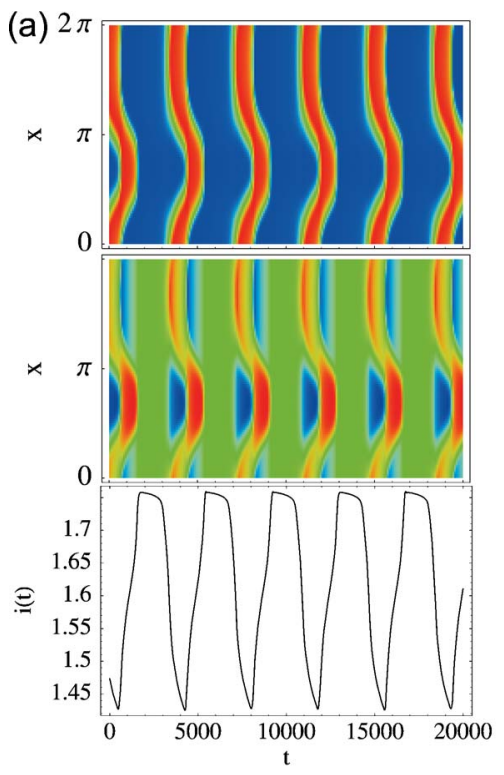

(b)

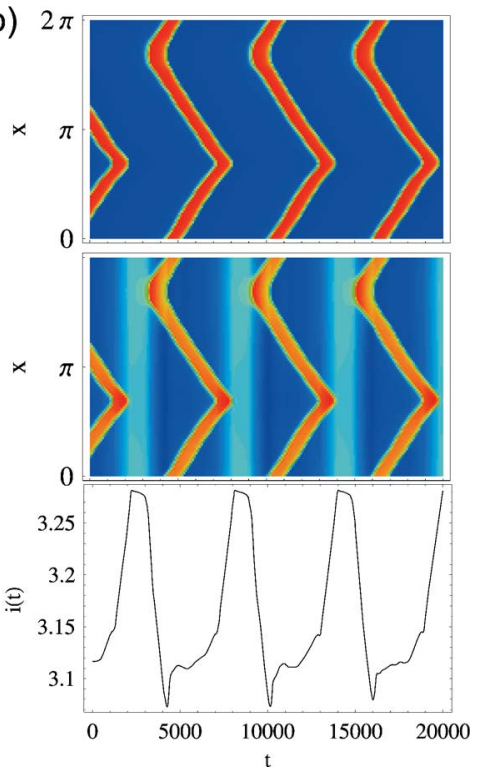

$h(x):=\left(\int_{t}^{t+T} \int_{0}^{\pi} i(x, t) d x d t\right)^{-1}$
FIG. 8. (Color online) Two examples of type-1 target patterns (TPs). Upper plates, $\phi_{\mathrm{DL}}(x, t)$; middle plates, $\phi_{\mathrm{DL}}-\left\langle\phi_{\mathrm{DL}}\right\rangle$; lower plates, $i(t)$. (a) Typical TP observed for most $U$, $\rho$, and $p_{\mathrm{Cu}}$ values. Parameters: $U=12, p_{\mathrm{Cu}}=p_{\mathrm{Cu}}^{0}$, $\rho=-0.9$. (b) TP as observed for larger aspect ratios (smaller $\beta$ ). Parameters: $U=11, \beta=0.1$, $\sigma=0.03, p_{\mathrm{Cu}}=p_{\mathrm{Cu}}^{0} \times 10^{-4}, \rho=-0.5$. For other parameters see Table I. 
(a)
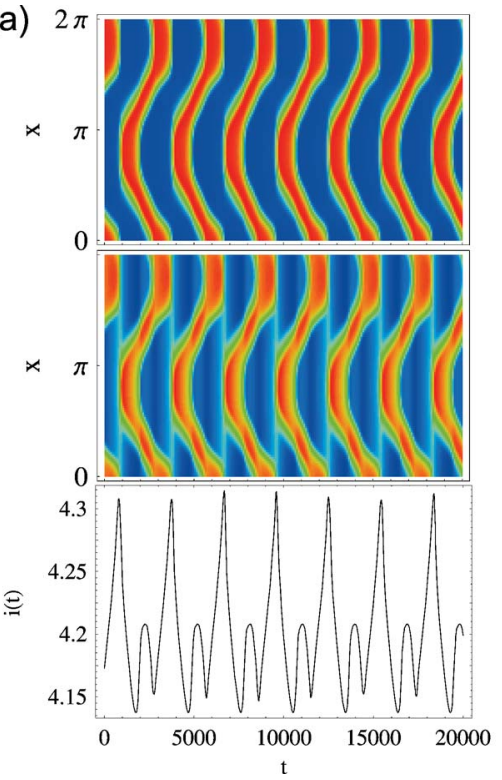

$$
\left(\int_{t}^{t+T} i(x, t) d t-\int_{t}^{t+T} \int_{0}^{\pi} i(x, t) d x d t\right)
$$

(b) $2 \pi$

$\times \pi$

$$
0
$$$$
\times \pi
$$

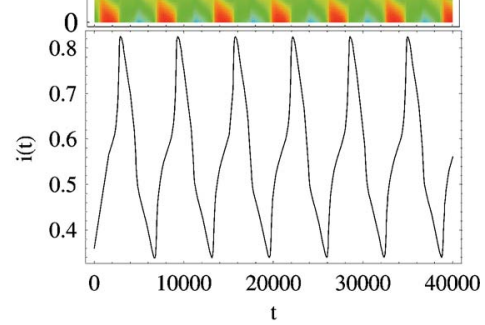
(s.b.).
FIG. 9. (Color online) (a) Second type of target pattern found during the HOR. Parameters: $U=15, p_{\mathrm{Cu}}=p_{\mathrm{Cu}}^{0} \times 10^{-2}, \rho=-0.9$. (b) Type- 3 target pattern observed at the lower $U$ boundary. Parameters: $U=3, p_{\mathrm{Cu}}=p_{\mathrm{Cu}}^{0} \times 10^{-4}, \rho=-0.7$. For other parameters see Table I. Upper plates, $\phi_{\mathrm{DL}}(x, t) ;$ middle plates, $\phi_{\mathrm{DL}}-\left\langle\phi_{\mathrm{DL}}\right\rangle$; lower plates, $i(t)$. could be defined to classify patterns with $r=\max _{x} h(x)<r_{t}$ as modulated oscillations and the ones above this threshold as target patterns. A systematic study of the evolution of $r$ as a function of the model parameters could not be performed due to the long integration times.

The passivation of the source point, which actually would be better characterized as a source region in this case, is accompanied by a steep fall in the total current density. The current minimum is reached as soon as the two waves meet on the opposite side of the ring. The active current plateau is reached after the annihilation.

For $\rho=-0.9, p_{\mathrm{Cu}}=p_{\mathrm{Cu}}^{0} \times 10^{-4}$, and $U>10$ the rising flank is interrupted by a small peak. The small peak continuously grows with increasing voltage until a qualitatively new form of target pattern as depicted in Fig. 9(a) is observed. The type-2 target pattern almost completely lacks the abovementioned asymmetry in time. The source and annihilation region are of the same size. The growth of the "prepeak" is accompanied by a shortening of the active period. Finally no active period remains and the two waves annihilate at the same time at which two new waves are sent out.

Figure 9(b) depicts a third type of a target pattern that is identified at the lower voltage boundary of oscillatory behavior. Again no active quasihomogeneous period is present. Despite the substantial similarity in the spatiotemporal pictures of the target pattern type-2 and the target pattern type- 3 the total current density reveals that they are indeed quite different. Target pattern type-3 almost resembles a cluster pattern since the annihilation region becomes active as soon as the source region goes passive. Target pattern type- 3 was classified as a target pattern rather than a cluster pattern for continuity reasons.

Target pattern type- 1 tends to coexist with the asymmetric target pattern. Note that in case the target pattern type-1 exhibits a small prepeak it is indistinguishable from an asym- metric target pattern on the basis of the global time series

\section{Asymmetric target patterns (A-TPs)}

Asymmetric target patterns represent oscillatory patterns in which two waves originate at a source point but only one of them travels around the ring. The other one is pinned at a certain position close to the wave source and stays approximately at this position until it annihilates with the wave emitted in the opposite direction [16]. Asymmetric target patterns have been presented in an earlier publication and are thus not discussed in detail at this point [24].

Regular asymmetric target patterns coexist with chaotically modulated pulses of the type shown in Fig. 6(a) in certain parameter regimes. On the other hand, regular asymmetric target patterns also coexist with target patterns of type-1 in different regions in parameter space. As can be expected due to the presence of a strong spatial defect in the asymmetric target pattern, asymmetric target patterns are favored for initial conditions also exhibiting such an inhomogeneity. Nevertheless, regular asymmetric target patterns can also be observed starting from homogeneous initial conditions.

Apart from regular asymmetric target patterns also irregular asymmetric target patterns are found (cf. [24]).

\section{E. Cluster patterns (CPs)}

The last building block of pattern formation induced by NGC during the HOR are Cluster patterns. Cluster patterns are characterized by the presence of a small number of domains of equal oscillation phase and amplitude. Two examples of cluster patterns found are presented in Fig. 10. The phase relation between two neighboring domains stays constant in time. Since the amplitudes of the individual oscillations in each domain are of the same size, cf. Fig. 11, these cluster patterns are termed phase clusters. 

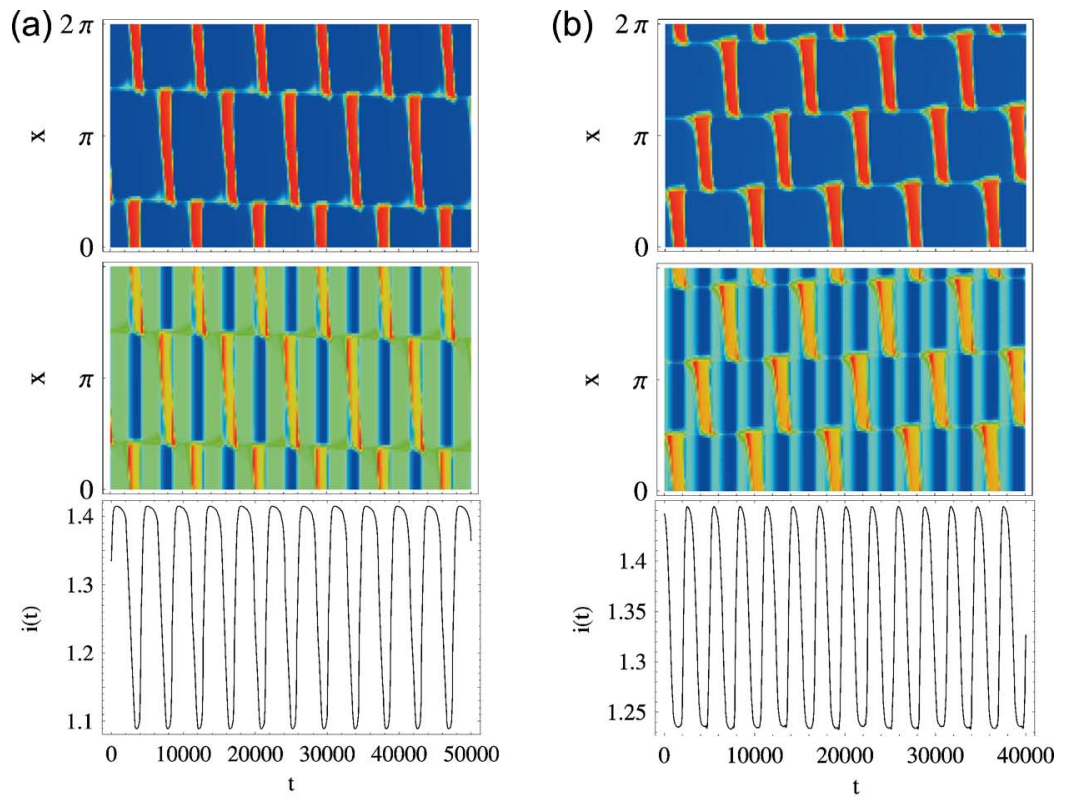

FIG. 10. (Color online) (a) Traveling twophase cluster pattern. (b) Traveling three-phase cluster pattern observed for the same parameter values. Upper plates, $\phi_{\mathrm{DL}}(x, t)$; middle plates, $\phi_{\mathrm{DL}}-\left\langle\phi_{\mathrm{DL}}\right\rangle$; lower plates, $i(t)$. (c) $\phi_{\mathrm{DL}}(x, t)$ at $t=21000$ and (d) at $t=15200$ for the cluster patterns presented in (a) and (b), respectively. $\mathrm{Pa}$ rameters: $U=5, \quad p_{\mathrm{Cu}}=p_{\mathrm{Cu}}^{0} \times 10^{-4}, \rho=-0.9$, for other parameters see Table I.
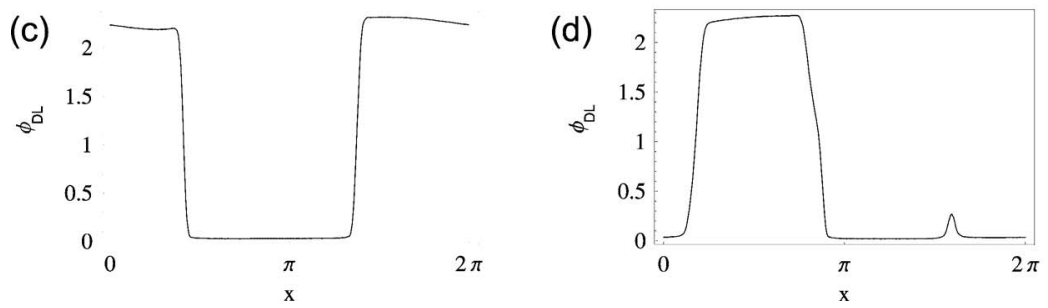

The phase domains are connected by sharp transition fronts. Since the oscillations are relaxationlike, the amplitudes of the different phase clusters are almost identical at certain stages of a full oscillatory cycle. During these active intervals the phase clusters are separated by a slightly more passive potential "wall." In all observed cluster patterns the fronts were not stationary in time but traveling with a constant velocity. Due to work by Ising and Bloch, traveling fronts are also called Bloch fronts [25]. The symmetry breaking instability leading to traveling fronts is called IsingBloch bifurcation.

The global time series and thus the spatiotemporal motion are found to be periodic in all cluster patterns.

In Fig. 10(a) a cluster pattern consisting of two phase domains is presented. The oscillatory medium is divided into two regions of equal size, as is clearly visible in Fig. 10(c) in

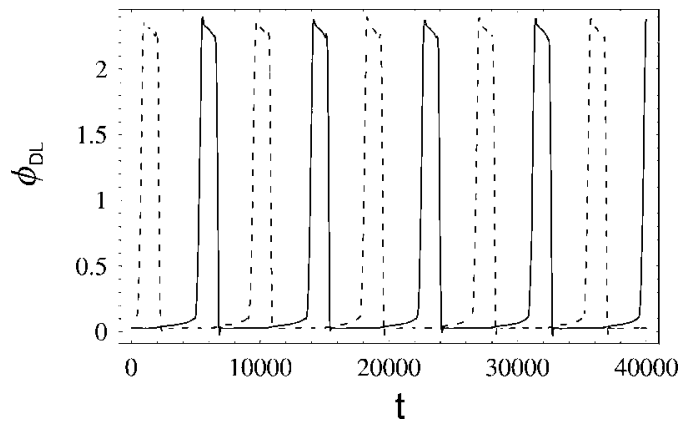

FIG. 11. Time series $\phi_{\mathrm{DL}}\left(x_{1 / 2}, t\right)$ at two different points $x_{1 / 2}$ in space representing the two-phase domains for the cluster pattern presented in Fig. 10(a). which a snapshot of the spatiotemporal motion at a certain time moment of the evolution is presented. This property is also called phase balance. It is a well-known characteristic of cluster behavior in reaction-diffusion systems and reflects the absence of an intrinsic length scale.

Apart from the two-phase cluster pattern, three-phase cluster patterns are also observed, actually the three-phase cluster pattern shown in Fig. 10(b) coexists with the twophase cluster pattern discussed above. The three-phase cluster pattern displays the same properties as the two-phase cluster pattern. Only Bloch fronts are stable and the phase balance is reached after transients decayed, cf. Fig. 10(d).

\section{DISCUSSION}

The local dynamics of the HOR model (1)-(4) exhibits relaxation oscillations in wide parameter regions, in agreement with experiments. In the present study the impact of a NGC on the spatiotemporal dynamics of an extended electrode during the oscillatory HOR was numerically investigated to help us understand experimental findings. However, from a more abstract point of view, we can consider the investigation also as a study of the dynamics of a field of relaxation oscillators that is subject to (non)local synchronizing and global desynchronizing coupling. Before comparing the results with the experiments, we discuss them from this more general point of view. The three two-parameter bifurcation diagrams shown in Figs. 2-4 reveal that the system exhibits a clear trend in response to the GC with increasing strength $|\rho|$. First of all, without NGC the entire system oscillated for all parameter values synchronously. Adopting the 
nomenclature used in connection with reaction diffusion (RD) systems to systems with nonlocal coupling, we can classify our reaction-migration system without $\mathrm{GC}$ as Benjamin Feir (BF) stable for all investigated conditions [26]. The spatially uniform oscillations remain stable for low GC strengths. By increasing the strength of the global coupling, the homogeneous limit cycle becomes unstable and is replaced by modulated oscillations with spatial variations developing on the steep flanks of the oscillations. Upon a further increase of the NGC strength, the spatial modulations become more pronounced, modulated oscillations transforming into target patterns, which, at the highest GC strength investigated tend to coexist with other inhomogeneous states, such as their asymmetric variant, pulses or cluster patterns, or they are replaced by one of the latter states. Thus, clearly, NGC favors the formation of inhomogeneous states also for BF stable relaxation oscillations, though, the resulting patterns possess for moderate coupling strength still quasihomogeneous intervals. Furthermore, the critical coupling strength at which oscillations are destabilized is considerable. Another type of electrochemical oscillators, so-called N-NDR (N-shaped negative differential resistance) oscillators [27] possess at the upper limit of the oscillatory region a large $U$ interval with harmonic oscillations, which only become relaxationlike close to the end of the oscillatory region at low $U$ values. In this system, inhomogeneous oscillations that are born in a wave bifurcation are found already at much lower values of the NGC strength [28]. Furthermore, when the oscillations are relaxationlike, spatial inhomogeneities predominantly develop when the current exhibits fast changes, similar to the modulated oscillations described above. It is likely that these findings do not depend on the specific systems but that quite generally spatial patterns develop at a lower strength of a NGC in the case of harmonic oscillations than for relaxation oscillations.

Next, let us discuss how the numerical results compare with experiments. First of all, all the patterns reported above, i.e., modulated oscillations, target patterns, asymmetric target patterns, pulses, and cluster patterns, were also observed experimentally $[16,29,30]$. In this context it is worth noting that even more subtle features of the dynamics are captured, such as the form of the current oscillations accompanying target patterns, including the fingerprint of the decelerated waves, or the double peak structure of the current time trace seen in case of the A- TPs $[16,24]$. Furthermore, also in experiments, pulses never had a constant shape or speed, but were modulated instead. However, in the case of the experimental pulses, the modulation frequency typically coincided with the time of circulation of the pulse. As discussed in [16], there are arguments supporting the hypothesis that inhomogeneities of the electrode surface lead to the pinning of the modulation frequency to the circulation frequency. But it could not be excluded that the modulations stem from surface inhomogeneities along the ring electrode and, hence, do not originate from an intrinsic dynamic instability. In the present numerical study, modulated pulses were found exclusively. This represents a further strong argument for the first conjecture.

The above cited experiments have recently been supplemented by a systematic study in the $\rho-U$-parameter plane

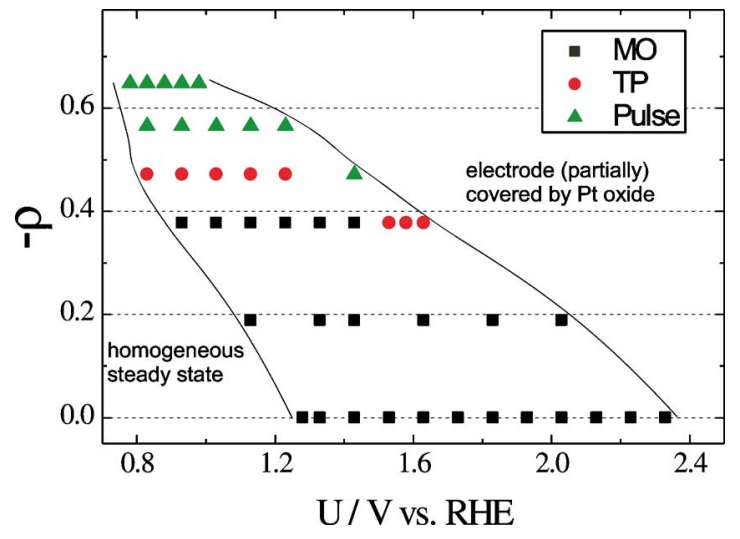

FIG. 12. (Color online) Experimentally obtained bifurcation diagram. Experimental details can be found in [29]. RHE, reversible hydrogen electrode [31].

for a $\mathrm{Cu}^{2+}$ concentration slightly higher than the one used in [16]. The results are depicted in Fig. 12. First, the basic building blocks of the bifurcation diagrams of Figs. 2-4, modulated oscillations, target patterns, and pulses, are again found in the experiments, target patterns occurring at higher values of $|\rho|$ than modulated oscillations and modulated pulses at higher $|\rho|$ than target patterns, which clearly matches the numerically found trend. However, there are also decisive differences, most importantly, modulated oscillations, similar to the ones depicted in Fig. 7, are already found at vanishing coupling strength, and also the other patterns are shifted to lower absolute values of $\rho$ compared with the simulations. Hence, the experiments suggest that the system is already BF unstable without GC. Indeed, at much lower $\mathrm{Cu}^{2+}$ concentration, a transition from modulated oscillations to turbulence was found for increasing $U$ and in the absence of GC [32]. In this parameter region, NGC destroyed the modulated oscillations already at coupling strengths much lower than in the present case and typically lead to clustered states rather than target patterns or pulses [30]. So far, BF unstable states were not obtained in the simulations, suggesting that at low $\mathrm{Cu}^{2+}$ concentrations there are elements in the local dynamics that are not captured in the model, or that there is a shift of one (or perhaps even more) parameters between model and experiment. This point calls for further investigations. Still, the qualitative agreement of the dynamics at high $\mathrm{Cu}^{2+}$ concentration substantiates our above discussion on the impact of NGC on relaxation oscillations.

\section{CONCLUSIONS}

Numerical investigations of spatiotemporal pattern formation during the oscillatory oxidation of hydrogen in the presence of $\mathrm{Cu}^{2+}$ and $\mathrm{Cl}^{-}$have been presented. The results given here present the study of the influence of negative GC on a relaxation oscillator. The reduced four variable model introduced in [17] was used for the modeling. The system displays homogeneous relaxation oscillations without global coupling for almost all parameter values tested. The existence of pulses was reported in a region of smaller aspect 
ratios in the absence of negative GC in the oscillatory regime.

With increasing NGC four distinct spatiotemporal patterns are observed. Pulses and antiphase oscillations and modulated oscillations and target patterns are found for intermediate negative GC. Additionally, we present the observation of cluster patterns and asymmetric target patterns at high negative GC. The first theoretical evidence of cluster patterns on a spatially extended electrode was given.

The theoretical results reproduce the experimentally observed patterns with outstanding consilience. Thus, it was shown that not only the homogeneous dynamics is captured by the model Eqs. (1)-(4), but that the reduced model also is sufficient to describe the rich previously uncaptured spatiotemporal dynamics during the HOR in the presence of negative GC. Besides the GC the only spatial coupling mechanism taken into account is the migration coupling acting on $\phi_{\mathrm{DL}}$. Again, we note that some trends in the experiments could not yet be reproduced and that the NGC strengths used in the simulations exceed those in the experiments.

The presented model can now be used for extended investigations of pattern formation during the HOR. Especially the influence of distributed parameters on the front motion in asymmetric target patterns and the modulation frequency of the modulated pulses is an interesting question. Also the introduction of a "toy model" that preserves the phenomenological spatiotemporal behavior but lacks the numerical difficulties would be desirable to gain deeper insight into the dynamics by computing bifurcation diagrams. A second unresolved issue is the turbulent dynamics observed in experiments without global coupling [32].

\section{ACKNOWLEDGMENT}

Financial support of the Deutsche Forschungsgemeinschaft in the framework of the Sonderforschungsbereich 555 "Complex Nonlinear Processes", Project No. B4, is gratefully acknowledged.

\section{APPENDIX}

Equations (1)-(4) are obtained from the reduced four variable model via the transformations

$$
\begin{gathered}
\phi_{\mathrm{DL}} \rightarrow \phi_{\mathrm{DL}}^{\prime}=\left|a_{\mathrm{Cl}}\right| \phi_{\mathrm{DL}}, \\
c \rightarrow c^{\prime}=c / c_{\mathrm{Cu}}^{b}, \\
t \rightarrow t^{\prime}=2 D_{\mathrm{Cu}} \delta_{\mathrm{Cu}}^{-2} t, \\
x \rightarrow x^{\prime}=(2 \pi L)^{-1} x,
\end{gathered}
$$

TABLE II. Physical constants.

$c_{x}^{b}=$ bulk concentration of species $x$

$N=$ number of free surface sites

$k_{x}^{a / b}=$ adsorption and desorption velocity of species $x$, respectively

$D_{x}=$ diffusion constant of species $x$

$a_{x}=$ potential dependence of the adsorption and/or desorption isotherm

$C_{D L}=$ capacity of the double layer

$\delta_{x}=$ thickness of the diffusion layer of species $x$

$\phi_{\mathrm{DL}}^{x, 0}=$ equilibrium voltage of adsorption and/or desorption isotherm of species $x$

$\sigma=$ specific conductivity of the electrolyte

$L=$ length of the electrode

$w=$ distance between $\mathrm{CE}$ and $\mathrm{WE}$

$R_{e}=$ external resistance

$R_{\Omega}=$ cell resistance

$R_{u}=$ uncompensated cell resistance, i.e., resistance between the RE and the WE

$U=$ applied voltage

$$
z \rightarrow z^{\prime}=z / w
$$

All voltages are rescaled accordingly. Primes are omitted in Eqs. (1)-(4) for clarity. The new parameters used are composed of the old one as follows (again primes were omitted at the obvious places). Definitions of the physical constants are given in Table II.

$$
\begin{gathered}
\nu=2 N\left(\delta_{\mathrm{Cu}} c_{\mathrm{Cu}}^{b}\right)^{-1}, \\
\tau_{\mathrm{Cl}}=2 D_{\mathrm{Cu}} N e^{-a_{\mathrm{Cl}} \phi_{\mathrm{DL}}^{\mathrm{Cl}, 0}\left(\delta_{\mathrm{Cu}}^{2} c_{\mathrm{Cl}}^{b} k_{\mathrm{Cl}}^{a}\right)^{-1},} \\
\tau_{\mathrm{Cu}}=2 D_{\mathrm{Cu}} N e^{-a_{\mathrm{Cu}} \phi_{\mathrm{DL}}^{\mathrm{Cu}, 0}\left(\delta_{\mathrm{Cu}}^{2} c_{\mathrm{Cu}}^{b} k_{\mathrm{Cu}}^{a}\right)^{-1},} \\
p_{x}=k_{x}^{d} e^{-2 a_{x} \phi_{\mathrm{DL}}^{x, 0}\left(k_{x}^{a} c_{x}^{b}\right)^{-1},} \\
\sigma \rightarrow \sigma^{\prime}=\frac{\sigma \delta_{\mathrm{Cu}}^{2}}{L C_{D L} D_{\mathrm{Cu}}(1+\rho)}, \\
\beta=2 \pi L w^{-1}, \\
a_{x} \rightarrow a_{x}^{\prime}=a_{x} / a_{\mathrm{Cl}}, \\
\rho=\frac{R_{e}}{R_{\Omega}}-\frac{R_{\Omega}-R_{u}}{R_{\Omega}} .
\end{gathered}
$$

Parameter values as obtained from the constants and parameters given in [17] are given in Table I. 
[1] G. Veser, F. Mertens, A. Mikhailov, and R. Imbihl, Phys. Rev. Lett. 71, 935 (1993).

[2] M. Falcke and H. Engel, Phys. Rev. E 50, 1353 (1994).

[3] A. von Oertzen, H. Rotermund, A. Mikhailov, and G. Ertl, J. Phys. Chem. B 104, 3155 (2000).

[4] E. Schöll, Nonlinear Spatio-Temporal Dynamics and Chaos in Semiconductors, Cambridge Nonlinear Science Series Vol. 10 (Cambridge University Press, Cambridge, 2001).

[5] Y. P. Raizer, Gas Discharge Physics (Springer, Berlin, 1997).

[6] K. Krischer, in Advances in Electrochemical Science and Engineering, edited by R. C. Alkire and D. M. Kolb (WileyVCH, New York, 2003), p. 89.

[7] V. Barelko, I. I. Kurochka, A. G. Merzhanov, and K. G. Shkadinskii, Chem. Eng. Sci. 33, 805 (1977).

[8] G. Philippou, F. Schultz, and D. Luss, J. Phys. Chem. 95, 3224 (1991).

[9] A. Winfree, The Geometry of Biological Time, 2nd ed. (Springer, New York, 2001).

[10] D. Golomb and J. Rinzel, Physica D 72, 259 (1994).

[11] V. K. Vanag, Y. Lingfa, M. Dolnik, A. M. Zhabotinsky, and I. R. Epstein, Nature (London) 406, 389 (2000).

[12] M. Kim, M. Bertram, M. Pollmann, A. Oertzen, A. S. Mikhailov, H. H. Rotermund, and G. Ertl, Science 292, 1357 (2001).

[13] J. Christoph, R. D. Otterstedt, M. Eiswirth, N. I. Jaeger, and J. L. Hudson, J. Chem. Phys. 110, 8614 (1999).

[14] J. Christoph, Ph.D. thesis, FU Berlin (1999), http:// www.diss.fu-berlin.de/2000/11.

[15] J. Christoph and M. Eiswirth, Chaos 12, 215 (2002).

[16] P. Grauel, H. Varela, and K. Krischer, Faraday Discuss. 120, 165 (2001).

[17] F. Plenge, H. Varela, M. Lübke, and K. Krischer, Z. Phys. Chem. 217, 365 (2003)

[18] K. Krischer, H. Varela, A. Birzu, F. Plenge, and A. Bonnefont, Electrochim. Acta 49, 103 (2003).
[19] N. Mazouz, K. Krischer, G. Flätgen, and G. Ertl, J. Phys. Chem. B 101, 2403 (1997).

[20] V. Stamenković and N. M. Marković, Langmuir 17, 2388 (2001).

[21] G. Flätgen and K. Krischer, J. Chem. Phys. 103, 5428 (1995).

[22] A. C. Hindmarsh, ACM SIGNUM Newsl. 15, 10 (1980), lsode is available from the netlib library http://www.netlib.org/ odepack/.

[23] N. Mazouz, G. Flätgen, and K. Krischer, Phys. Rev. E 55, 2260 (1997).

[24] F. Plenge, H. Varela, and K. Krischer, Phys. Rev. Lett. 94, 198301 (2005).

[25] P. Coullet, J. Lega, B. Houchmanzadeh, and J. Lajzerowicz, Phys. Rev. Lett. 65, 1352 (1990).

[26] Y. Kuramoto, Chemical Oscillations, Waves and Turbulence, Springer Series in Synergetics Vol. 19, edited by H. Haken, (Springer, Berlin, 1984).

[27] K. Krischer, in Modern Aspects of Electrochemistry, edited by B. E. Conway, J. O. Bockris, and R. White (Kluwer Academic/ Plenum, New York, 1999), Vol. 32, p. 1.

[28] F. Plenge, Y.-J. Li, and K. Krischer, J. Phys. Chem. B 108, 14255 (2004).

[29] H. Varela, Ph.D. thesis, FU Berlin (2003), http://darwin.inf.fuberlin.de/2003/304/.

[30] H. Varela, C. Beta, A. Bonnefont, and K. Krischer, Phys. Chem. Chem. Phys. 7, 2429 (2005).

[31] C. H. Hamann, A. Hamnett, and W. Vielstich, Electrochemistry (Wiley-VCH, Weinheim, 1998).

[32] H. Varela, C. Beta, A. Bonnefont, and K. Krischer, Phys. Rev. Lett. 94, 174104 (2005).

[33] Chaotic dynamics are assumed since no periodicity was observed during $\sim 500$ oscillations periods.

[34] Note that these target patterns are not connected to excitable dynamics or inhomogeneities on the ring. 